\title{
Textile-based sensor systems for condition monitoring in composite and medical applications
}

\author{
Andreas Nocke, Eric Häntzsche, Johannes Wendler, Chokri Cherif \\ Institute of Textile Machinery and High Performance Material Technology (ITM) at Technische \\ Universität Dresden, 01062 Dresden, Germany \\ Corresponding e-mail address: Andreas.Nocke@tu-dresden.de
}

\begin{abstract}
Textile-based sensors benefit from their high flexibility, low production cost and ability to monitor large scaled components making them particularly suitable for special application scenarios. For example, they are well suited for the monitoring of structural or human health states enabling the detection of mechanical stresses of composite components or physiological parameters in medical applications. Textile-based sensors can be integrated automatically into woven, knitted or non-crimp fabrics. Using these manufacturing techniques tailored simple one-dimensional or complex two-dimensional sensor layouts can be realized. By combining individual sensors to sensor networks, multiple parameters are detectable simultaneously.
\end{abstract}

Key words: Textile-based sensor, condition monitoring, functionalized fabric, FRP, wound dressing

\section{Introduction}

In recent years, textile approaches have opened up new fields of application besides conventional clothing textiles. Especially for lightweight composite materials like fibre reinforced plastics (FRP), textile solutions have gained significant importance as an alternative for conventional materials, like steel and aluminum. In medical engineering, the applications range of technical textiles comprises amongst others antimicrobial surgical textiles, wound dressings, resorbable yarns for sewing of internal wounds as well as tissue engineering of artificial implants on a textile basis (e. g. vascular stents or meshes for the treatment of inguinal hernias).

Condition monitoring in general and particularly in textile based products enables significant advantages: (i) improved fundamental understanding of the material and structural properties, (ii) active feedback loops are usable to precisely adjust process or material parameters and (iii) economic advantage for companies due to added value and/or reduced failure risks. With respect to textile-based materials condition monitoring using textilebased sensors are often favorable compared to other conventional sensor systems due to the almost identical mechanical and chemical properties as well as the capability to use the same processing tools. The combination of textile-based carrier and/or reinforcement materials with textile-based sensors results in functionalized materials with high flexibility, low production cost and a scalability allowing e.g. the monitoring of large FRP-components. Obviously, textile-based sensors are to a certain degree limited in terms of complex setups with integrated electronics as they are known in microelectromechanical systems. However, textile processes allow the highly automated fabrication of multi layered yarn structures with specific electrical, chemical and mechanical properties of each layer. Using such complex rotationally symmetric structures, sensors are available, which are based amongst others on the resistive, piezoelectric, impedimetric or fibre-optic transducer mechanism (Fig. 1).

a)

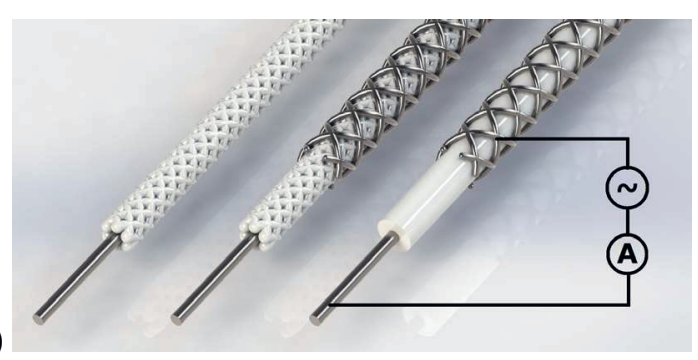




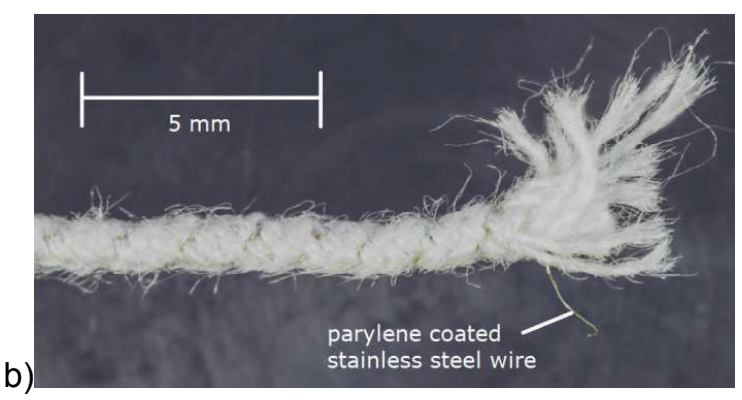

Fig. 1. Complex textile-based sensor structures: Coaxial sensor set-up for impedimetric measurement (a) and strain-relieved braided helical sensor structure for temperature measurement (b)

\section{Integration techniques for textile-based sensors}

In general, most textile processing techniques, like weaving, warp or flat knitting respectively, are suitable to integrate additional functional yarns. Main limitations can result from the capability to withstand process related yarn tensions and critical bending radii of the functional yarns. With respect to the latter fibreoptic based transducers give an example for a reduced textile processing compatibility.

Depending on the textile process yarn directions are predefined for conventional machines and therefore restrict possible layouts for textile based sensors and sensor networks. Especially for mechanical sensors designed to detect loads or structural degradations, a component specific orientation and positioning of each sensor is important for accurate measurement systems.

One way to achieve this goal is to use the embroidering process with the Tailored Fibre Placement (TFP) technique. TFP is a machinecontrolled application technology, by which the additional functional yarn is stitched onto the textile fabric in a subsequent textile production process. However, such functional elements are not integral elements of the reinforced structure anymore. The advantage of the TFP technology is its layout variability, because functional yarns can be applied in any geometric form and orientation in fabric plane of almost any pre-produced textile fabric, e.g. nonwovens, spacer fabrics or non-crimp fabric.

Another technological approach to realize complex two-dimensional sensor layouts comprises the modification of conventional textile processes by adding so called yarn path manipulation devices. Going this way, a textiletechnological process is available being capable to integrate the sensor yarns fully automated and almost freely scalable. Additionally, the load transmission behavior between reinforcement and sensor yarn can be tailored to the specific demands. For multi-axial non-crimp fabrics (NCF) as one of the main textile reinforcement structures in FRP- and Carbon Concrete Composite-( $\left.\mathrm{C}^{3}\right)$ components, a warp yarn path manipulation (WPM) device is available, enabling the translational displacement of individual sensor yarn bands over the fabric's width (see Fig. 2). With respect to woven structures, a multi-axial weaving machine with Open Reed Weave (ORW) technology and similar WPM device for additional warp bands is presented. This innovative weaving machine allows the integration of textile-based sensors with custom multi-dimensional layouts during the weaving process (see Fig. 3).

a)

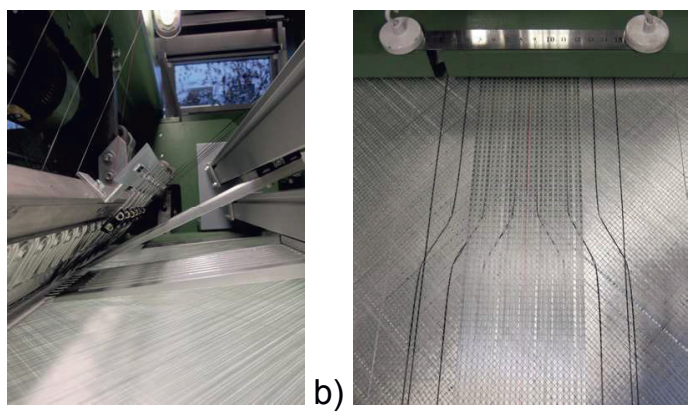

Fig. 2. WPM unit in MALIMO® 14024 knitting machine: laying device in front of the knitting area (a) and structurally integrated Carbon fibre yarn (CFY) strain sensors in glass fibre NCF (b)

a)

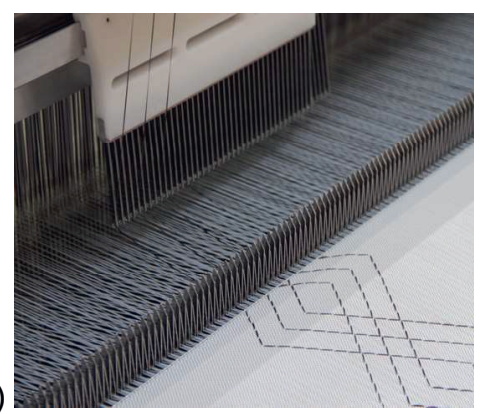

b)

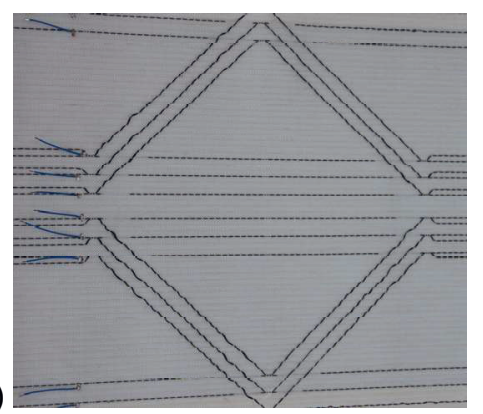

Fig. 3. Multi-axial weaving machine with Open Reed Weave (ORW) technology for yarn path modification: (a) ORW device and (b) structurally integrated CFY strain sensors in PES-based fabric for textile architecture applications 


\section{Structural health monitoring of fibre- reinforced composite components}

In order to advance lightweight design approaches while sustaining safety standards, the detection of serious structural damage at an early stage as well as a continuous in situ monitoring of local and global loads of composite components become increasingly necessary. The majority of conventional structural health monitoring (SHM) systems are still too costly and time-consuming in their installation as well as not optimised for the use in complex FRP components, e.g. wind turbine blades. Previous investigations have shown the suitability of carbon fibre yarn (CFY) acting as appropriate basic-material to be integrated as two-dimensional sensor for in situ load monitoring as well as condition monitoring of composites components [1-4]. Compared to commercially available Fibre Bragg Grating (FBG) sensors or conventional metal foil strain gauges, the CFY sensor approach is more adaptable to specific application requirements. In addition to the cost-intensive measurement equipment (e.g. interrogator for FBG sensor), the further textile-technological processing of FBG sensors is restricted due to the critical bending radii of the glass fibre monofil [5-6]. Compared to conventional strain, the major benefit of CFY sensors is both the considerable time- and cost-saving potential due to their fully automated textile-technological integration. Furthermore, they have an outstanding in situ measuring capability, which enables strain measurement with excellent gauge protection due to the surrounding polymer matrix, even in inaccessible areas within immediate distance of the load-bearing FRP reinforcement plies [7-8].

To verify the functional capability of structurally integrated textile-based CFY-sensors operating for condition and structural health monitoring systems, a functionalised glass fibre based FRP-demonstrator of a small wind turbine blade in thermoset composite design is developed by the authors (Fig. 4).

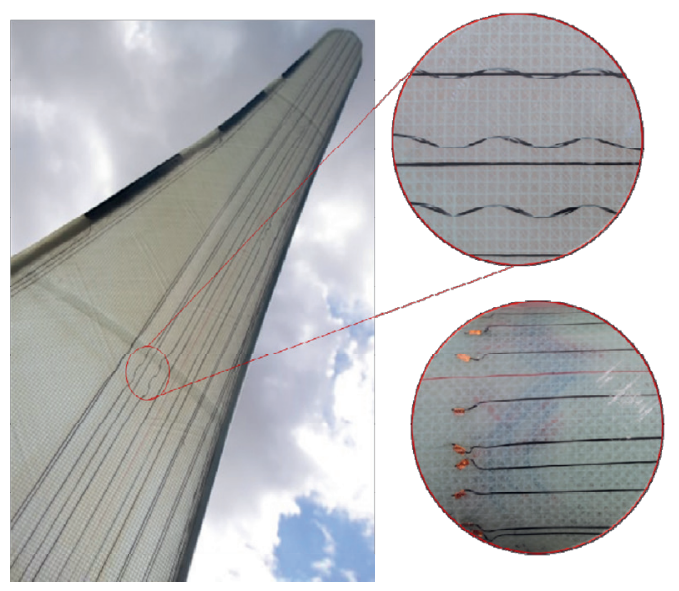

Fig. 4. Functional FRP-demonstrator of wind turbine blade with detailed view on electrical contacting and alignment of length-graded CFY sensors

To achieve the in situ strain measurement inside the wind turbine blade with spatial resolution capability, CFY sensors with different gauge lengths are designed for the integration in the outer textile reinforcement layer (triaxial NCF ply) that acts as tension respectively compression flanges of the rotor blade semi shells. The developed sensor network consists of several CFY circuits, each covering a different blade section (Fig. 5), thus enabling the spatially resolved measurement of the accumulated strain from the rotor blades' root up to its tip.

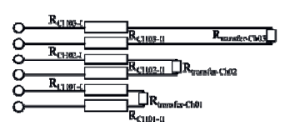

a)Equivalent circuit

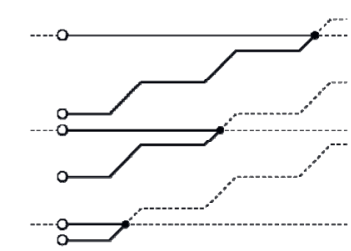

b) Multiaxial warp knitting with warp yarn path manipulation device

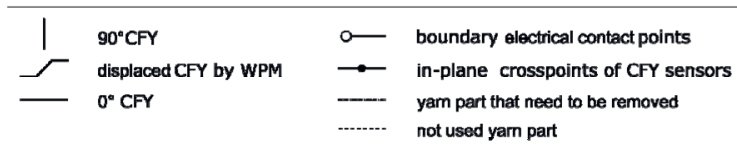

Fig. 5. Equivalent circuit of CFY sensors with different gauge lengths (a) and schematic of technological implementation of using WPM units (b)

The resulting sensor signals are analyzed systematically for varying load scenarios and compared to simulation results of the mechanical behaviour of the FRP-demonstrator by non-linear FEM modelling with LS-DYNA. As it can be seen in Fig. 6, a good correlation between applied loads, calculated strain and measured sensor signals is achieved. For further information, please refer to [3-4]. 


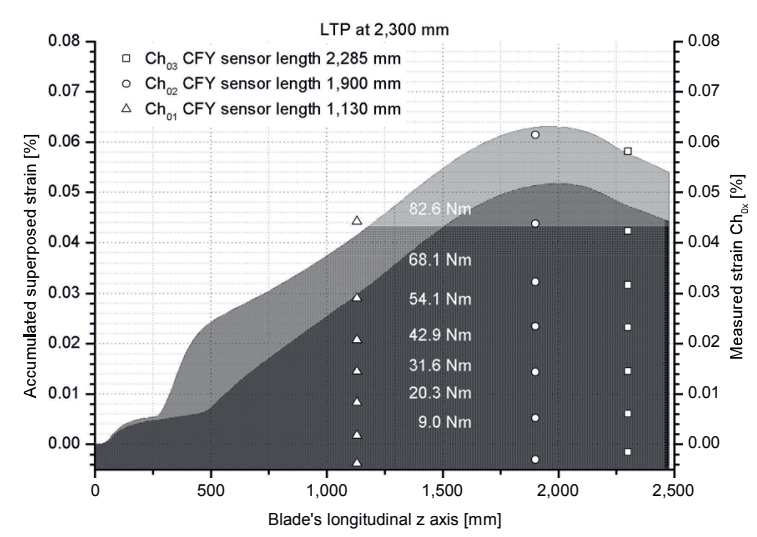

Fig. 6. Comparison of both simulated (grey value filled curves) and measured strain ( $\mathrm{Ch}_{01}$ to $\left.\mathrm{Ch}_{03}\right)$ with increasing bending loads from $9.0 \mathrm{Nm}$ up to $83 \mathrm{Nm}$ and load transmission point at 2,300 mm

\section{Monitoring of physiological indicators in medical applications}

The knowledge of physiological indicators plays a crucial role in various medical applications, like evaluation of the health state of high risk patients (e.g. diabetes), the monitoring of the healing process after traumatic injuries or of chronic wounds.

For last-mentioned application, specific physiological indicators are amongst others the temperature, $\mathrm{pH}$-value, lactate content or concentration of immune cells [9]. Various yarn structures and geometries, which are suitable for specific sensor methods can be manufactured by braiding. A helically integrated wire in a strain-relieved braided yarn enables a temperature measurement with high metrological stability under mechanical stresses.

With the use of functional, biocompatible materials like viscose or chitosan moisture or $\mathrm{pH}$-sensor yarns based on the coaxial sensor set-up for impedimetric measurements as shown in Fig. 1a) can be produced. The latter one is based on the swelling characteristics of chitosan, crosslinked into a hydrogel which is sensitive to different pH-values (Fig. 7).
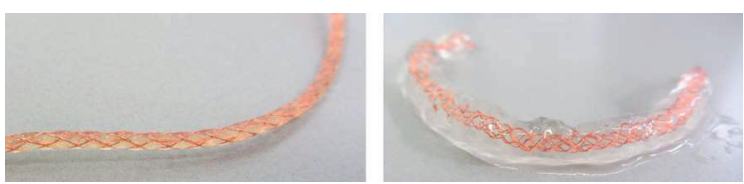

Fig. 7. Swelling characteristic of cross-linked chitosan in $\mathrm{pH} 7$ (left) and $\mathrm{pH} 5$ (right)

In Fig. 8 the impedance of the sensor yarns with functional chitosan layers are displayed in comparison to reference sensor constructions with layers of PTFE and viscose. The textile-based $\mathrm{pH}$ sensors with chitosan as functional layer exhibit a significant correlation to the given $\mathrm{pH}$ values.



Fig. 8. Braided $\mathrm{pH}$-sensors with chitosan layer as functional layer (upper blue curves) in comparison to braided reference sensors with PTFE and viscose layers (lower blue curves) for varying $\mathrm{pH}$-values (green curve)

\section{Conclusion}

Textile-based sensors are capable for the measurement of various mechanical, chemical and physiological parameters. For application scenarios, especially where textile carrier or reinforcement layers are used, they exhibit specific advantages over conventional sensor concept. These advantages origin mainly from the material compatibility in terms of flexibility and mechanical strength as well as the high automation level of textile processes. Especially yarn path manipulation devices allows the inprocess integration of customized 2D sensorlayouts for condition and SHM-monitoring of FRP-components and medical engineering. For the FRP-application production rates up to 16 $\mathrm{m} / \mathrm{min}$ have been tested successfully. The simultaneous fabrication of both textile reinforcement structure and in situ strain sensors within the subsequent load-dissipating area of FRP components has been successfully shown with the help of a small wind turbine blade. With respect to the medical engineering application specific textile-based sensors have been developed being able to identify important physiological parameters e. g. for monitoring of chronic wounds.

Especially in terms of long term stability and sensitivity, additional research work is required to establish textile-based sensors with a comparable feature profile as conventional sensors.

\section{Acknowledgements}

The IGF research projects $18640 \mathrm{BR} / 1$ and 18901 BR of the Forschungsvereinigung Forschungskuratorium Textil e. $V$. in cooperation with the Forschungsgesellschaft für Messtechnik, Sensorik und Medizintechnik e. V. is funded through the AiF within the program for supporting the "Industriellen Gemeinschaftsforschung (IGF)" from funds of the Federal Ministry of Economics and Energy 
(BMWi) by a resolution of the German Bundestag.

We also like to thank the German Research Foundation for funding the DFG project CH174/40-1.

\section{References}

[1] E. Haentzsche, A. Matthes, A. Nocke, Ch. Cherif, Characteristics of carbon fibre based strain sensors for structural-health monitoring of textilereinforced thermoplastic composites depending on the textile technological integration process. $J$ Sens. Actuators, A 203, 189-203 (2013)

[2] E. Haentzsche, B. Schneider, A. Nocke, G. Hoffmann, Ch. Cherif, Integrally manufactured sensor systems for structural health monitoring using ORW weaving technology. Technical Textiles 57, 175-176 (2014)

[3] E. Haentzsche, R. Mueller, M. Huebner, T. Ruder, R. Unger, A. Nocke, Ch. Cherif, Manufacturing technology of integrated textilebased sensor networks for in situ monitoring applications of composite wind turbine blades. Journal of Smart Materials and Structures 25, 105012, 11pp (2016) DOI: https://doi.org/10.1088/0964-1726/25/10/105012

[4] Ch. Cherif, E. Haentzsche, R. Mueller, A. Nocke, M. Huebner and M. M. B. Hasan: Carbon fibre sensors embedded in glass fibre-based composites for wind turbine blades. In: Smart Textiles and Their Applications. Woodhead Publishing Series in Textiles: Number 178. ISBN 978-1-84569-723-5. pp. 329-352

[5] K. Kim, J.M. Lee, Y. Hwang, Determination of engineering strain distribution in a rotor blade with fibre Bragg grating array and a rotary optic coupler, J Opt. Lasers Eng. 46, 758-762 (2008)

[6] A. Papantoniou, G. Rigas, N.D. Alexopoulos, Assessment of the strain monitoring reliability of fibre Bragg grating sensor (FBGs) in advanced composite structures. J Compos. Struct. 93, 2163-2172 (2011)

[7] J. Sebastian, N. Schehl, M. Bouchard, M. Boehle, L. Li, A. Lagounov, K. Lafdi, Health monitoring of structural composites with embedded carbon nanotube coated glass fibre sensors. Carbon 66, 191-200 (2014)

[8] N.D. Alexopoulos, C. Bartholome, P. Poulin, Z. Marioli-Riga, Structural health monitoring of glass fibre reinforced composites using embedded carbon nanotube (CNT) fibres. Compos. Sci. Technol. 70, 260-271 (2010)

[9] A. Nocke, A. Schröter, Ch. Cherif, G. Gerlach, Miniaturized textile-based multi-layer ph-sensor for wound monitoring applications. Autex Research Journal, 12(1), 20-22 (2012) 